\title{
Charm Decays at Belle
}

\section{Min-Zu Wang ${ }^{* i}$}

Department of Physics, Institute of Astrophysics and

Leung Center for Cosmology and Particle Astrophysics,

National Taiwan University, Taiwan, R.O.C.

E-mail: mwang@phys.ntu.edu.tw

Preliminary results of the charmed hadron decays from Belle are presented. The absolute branching fractions of leptonic $D_{s}$ decays $D_{s} \rightarrow \mu v$ and $D_{s} \rightarrow \tau v$ are measured to be $(0.528 \pm$ 0.028 (stat.) \pm 0.019 (syst. $)) \%$ and $\left(5.70 \pm 0.21_{-0.30}^{+0.31}\right) \%$, respectively. The $D_{s}$ decay constant $f_{D_{s}}$ is determined to be $255.0 \pm 4.2 \pm 4.7 \pm 1.8\left(\tau_{D_{s}}\right) \mathrm{MeV}$ which is consistent with the lattice QCD calculation. The Cabibbo suppressed charmed baryon decays, namely $\Xi_{c}^{0} \rightarrow \Xi^{-} K^{+}$and $\Xi_{c}^{0} \rightarrow \Lambda \phi$, are first observed. These results are obtained based on a large data sample collected by the Belle detector at the KEKB asymmetric energy $e^{+} e^{-}(3.5$ on $8 \mathrm{GeV})$ collider.

36th International Conference on High Energy Physics,

July 4-11, 2012

Melbourne, Australia

* Speaker.

${ }^{\dagger}$ on behalf of the Belle Collaboration 


\section{Introduction}

Measurements [1] of the absolute branching fractions of leptonic $D_{s}^{+}$[2] decay offer a clean probe to the decay constant of $D_{s}, f_{D_{s}}$, and the relevant Cabibbo-Kobayashi-Maskawa matrix element, $\left|V_{c s}\right|$. The Standard Model (SM) calculation renders the following equation:

$$
\mathscr{B}\left(D_{s}^{+} \rightarrow l^{+} v_{l}\right)=\frac{G_{F}^{2}}{8 \pi} f_{D_{s}}^{2}\left|V_{c s}\right|^{2} \tau_{D_{s}} M_{D_{s}} m_{l}^{2}\left(1-\frac{m_{l}^{2}}{M_{D_{s}}^{2}}\right)^{2},
$$

where $G_{F}$ is the Fermi coupling constant and $\tau_{D_{s}}$ stands for the lifetime of the $D_{s}^{+}$meson. A recent lattice QCD calculation [3] gives a very precise estimation of $f_{D_{s}}$ with an uncertainty at percent level. The above measurement is also sensitive to new physics beyond SM, e.g. two Higgs doublet model since the coupling to charged Higgs can give extra contribution to the decay width of $D_{s}^{+}$. It is without doubt that any effort on the experimental side to determine accurately the leptonic decay width of $D_{s}^{+}$is very important.

The weak decay mechanism of charmed baryons is less known than that of charmed mesons, especially for those Cabibbo-suppressed decays. The incidents are quite rare and there is few experimental information [1] to be useful in order to compare with theoretical predictions [4].

We use the full data set collected near the $\Upsilon(4 S)$ resonance by the Belle detector [5] at the KEKB asymmetric energy $e^{+} e^{-}(3.5$ on $8 \mathrm{GeV})$ collider [6] to search for rare $\Xi_{c}^{0}$ decays and use an even larger data set (adding $\Upsilon(5 S)$ data) to measure $\mathscr{B}\left(D_{s}^{+} \rightarrow l^{+} v_{l}\right)$. Preliminary results will be presented in the following sections.

\section{2. $D_{s}^{+} \rightarrow l^{+} v_{l}$}

We use the following process, $e^{+} e^{-} \rightarrow c \bar{c} \rightarrow \bar{D}_{t a g} K X_{\text {frag }} D_{s}^{*+}$ and $D_{s}^{*+} \rightarrow D_{s}^{+} \gamma$, to study the leptonic $D_{s}^{+}$decays. We select an energetic charmed hadron as $\bar{D}_{\text {tag }}$ which could be $\bar{D}^{0}, D^{-}, \Lambda_{c}^{-} p, D^{*-}$ or $\bar{D}^{* 0}$. The tagged charmed hadron is reconstructed by up to 6 dominant sub-decays. To balance the strangeness, we require $K$ to be either a charged kaon or a $K_{S}^{0}\left(\rightarrow \pi^{+} \pi^{-}\right)$meson. Only limited fragmentation remnants are considered. So $X_{\text {frag }}$ can be nothing, $\pi^{ \pm}, \pi^{0}, \pi^{ \pm} \pi^{ \pm}, \pi^{ \pm} \pi^{0} . \pi^{ \pm} \pi^{ \pm} \pi^{ \pm}$, or $\pi^{ \pm} \pi^{ \pm} \pi^{0}$. We use our particle identification system (PID) to identify charged kaon or pion. The low energy $\gamma$ from $D_{s}^{*+} \rightarrow D_{s}^{+} \gamma$ should have energy greater than $0.12 \mathrm{GeV}$ measured by the electromagnetic calorimeter (ECL) with reconstructed direction opposite to the $\bar{D}_{t a g}$ side. The inclusive $D_{s}$ signal is identified with the missing mass variable $M_{\text {miss }}\left(\bar{D}_{\text {tag }} K X_{\text {frag }} \gamma\right)=\sqrt{p_{\text {miss }}^{2}\left(\bar{D}_{\text {tag }} K X_{\text {frag }} \gamma\right)}$, where $p_{\text {miss }}=p_{e^{+}}+p_{e^{-}}-p_{\bar{D}_{\text {tag }}}-p_{K}-p_{X_{\text {frag }}}-p_{\gamma}$ is the missing 4-momentum in the event.

Figure 1 shows the inclusive $D_{s}$ signal with $X_{\text {frag }}=\pi^{ \pm}$. A peak at the mass of $D_{s}^{+}$is easily identified in the missing mass spectrum. We estimate that the total signal yield in the $7 X_{\text {frag }}$ cases is about 94400. Within this inclusive sample, we can search for exclusive decays of $D_{s}^{+}$meson. For example, $D_{s}^{+} \rightarrow \mu^{+} v_{\mu}$ can be identified by $M_{\text {miss }}\left(\bar{D}_{\text {tag }} K X_{\text {frag }} \gamma \mu\right)$ with a peak at zero since the neutrino mass is close to zero. The efficiency of the reconstructed exclusive signal given correct inclusive $D_{s}$ tag is estimated by Monte Carlo samples.

As a check on the above method, we perform the branching fraction measurements for $D_{s}^{+} \rightarrow$ $K^{+} K^{-} \pi^{+}, D_{s}^{+} \rightarrow K_{S}^{0} K^{+}$and $D_{s}^{+} \rightarrow \eta \pi^{+}$. The following figures 2-4 show the reconstructed signal peaks. The measured branching fractions are $\mathscr{B}\left(D_{s}^{+} \rightarrow K^{+} K^{-} \pi^{+}\right)=(5.06 \pm 0.15$ (stat. $) \pm$ 
0.19 (syst. $)) \%, \mathscr{B}\left(D_{s}^{+} \rightarrow \bar{K}^{0} K^{+}\right)=(2.84 \pm 0.12 \pm 0.08) \%$, and $\mathscr{B}\left(D_{s}^{+} \rightarrow \eta \pi^{+}\right)=(1.79 \pm 0.14 \pm$ $0.05) \%$. They are all in good agreement with the corresponding world average [1].

With the confidence on this method, we performed the leptonic branching fraction measurement for $D_{s}^{+} \rightarrow \mu^{+} v_{\mu}$ and $D_{s}^{+} \rightarrow \tau^{+} v_{\tau}$. The missing mass spectrum for $M_{\text {miss }}^{2}\left(\bar{D}_{\text {tag }} K X_{\text {frag }} \gamma \mu\right)$ is shown in Fig. 5. The measured branching fraction for $D_{s}^{+} \rightarrow \mu^{+} v_{\mu}$ is $(0.528 \pm 0.028 \pm 0.019) \%$. This is the most precise measurement by a single experiment up-to-date.

We reconstruct $\tau$ via the following three decay modes: $\tau \rightarrow e v \nu, \tau \rightarrow \mu \nu v$ and $\tau \rightarrow \pi \nu$. The $D_{s}^{+} \rightarrow \tau^{+} v_{\tau}$ signal is selected with $M_{\text {miss }}\left(\bar{D}_{\text {tag }} K X_{\text {frag }} \gamma\right)$ in the $D_{s}$ region and only one remaining track pointing to the interaction point (IP). The $e, \mu$, or $\pi$ should be positively identified by PID. After removing the matched clusters with known decay particles, we then check the remaining activity in ECL. The signal should peak at zero. The measured results for the above three modes and the combined result are listed in the following Table.

\begin{tabular}{lc}
\hline \hline$\tau$ decay mode & $\mathscr{B}\left(D_{s}^{+} \rightarrow \tau^{+} v_{\tau}\right)\left[\times 10^{-2}\right]$ \\
\hline \hline$e v v$ & $5.37 \pm 0.33_{-0.30}^{+0.35}$ \\
$\mu \nu v$ & $5.88 \pm 0.37_{-0.58}^{+0.34}$ \\
$\pi \nu$ & $5.96 \pm 0.42_{-0.39}^{+0.45}$ \\
\hline Combination & $5.70 \pm 0.21_{-0.30}^{+0.31}$ \\
\hline \hline
\end{tabular}

Using the obtained leptonic branching fractions of $D_{s}$, we can estimate $f_{D_{s}}$ and the result is $255.0 \pm 4.2 \pm 4.7 \pm 1.8\left(\tau_{D_{s}}\right) \mathrm{MeV}$. It agrees with the theoretical prediction [3] at $2 \sigma$ level.

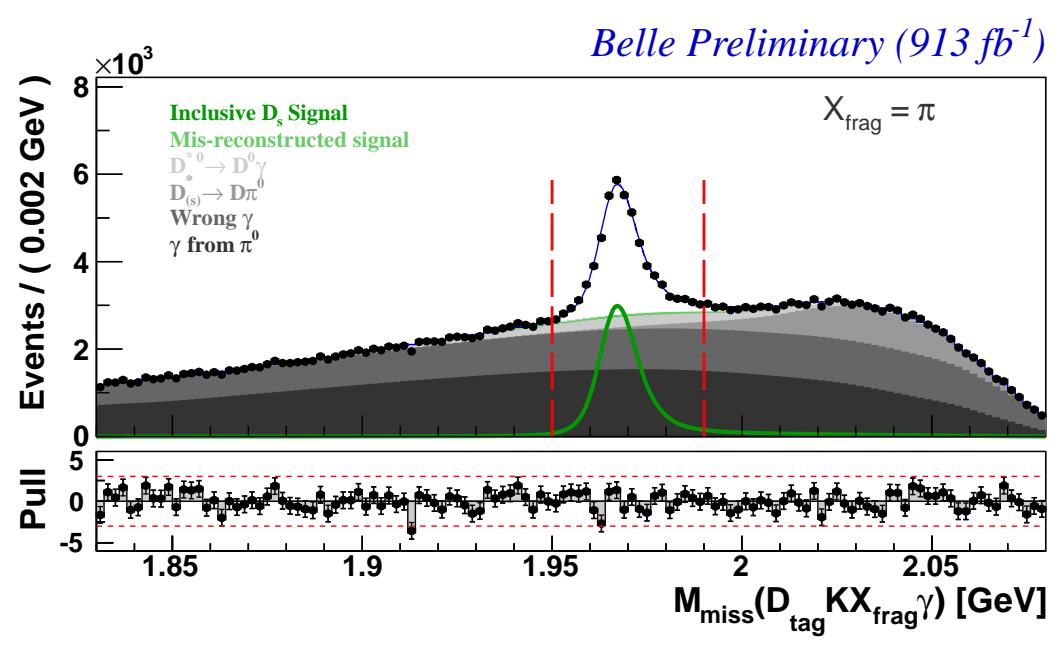

Figure 1: The $M_{\text {miss }}\left(D_{\text {tag }} K X_{\text {frag }}\right)$ distributions with $X_{\text {frag }}=\pi^{ \pm}$. Fit results are overlaid and pull distribution is attached. The inclusive $D_{s}$ signal is clearly seen.

\section{3. $\Xi_{c}^{0} \rightarrow \Xi^{-} K^{+}$and $\Xi_{c}^{0} \rightarrow \Lambda \phi$}

We search for the Cabibbo suppressed decays of the $\Xi_{c}^{0}$ baryon with the following final state particles, $\Lambda K^{+} \pi^{-}$and $\Lambda K^{+} K^{-}$. The light mesons should be positively identified by PID. The long lived $\Lambda$ is reconstructed with $\Lambda \rightarrow p \pi^{-}$. The $\Xi^{-}$Hyperion is selected by $\Xi^{-} \rightarrow \Lambda \pi^{-}$with its 


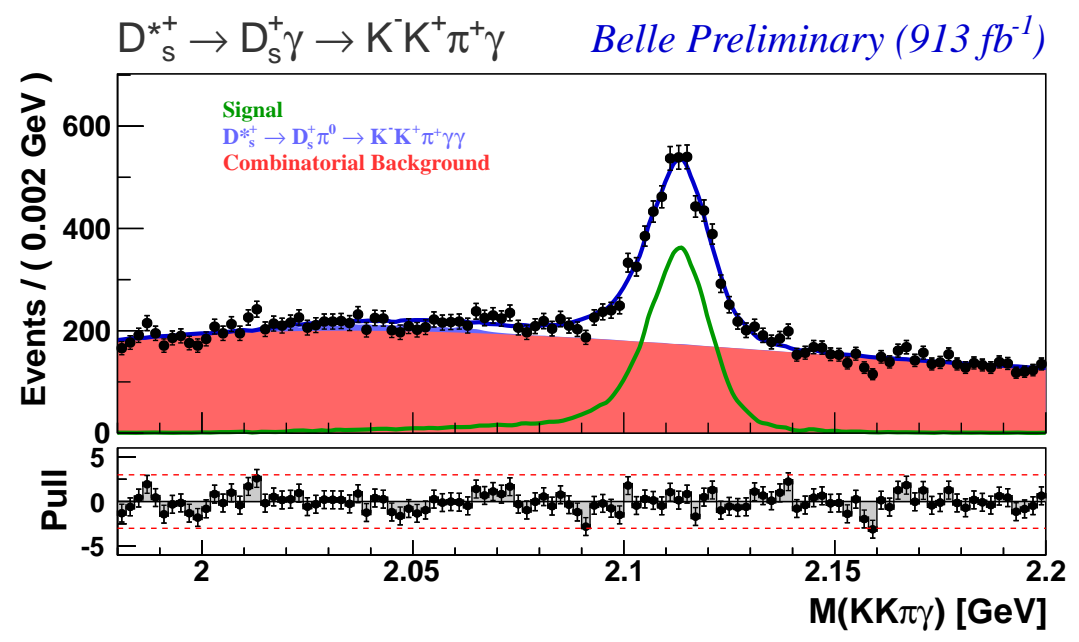

Figure 2: The $M\left(K^{+} K^{-} \pi^{+} \gamma\right)$ distribution of exclusively reconstructed $D_{s}^{+} \rightarrow K^{+} K^{-} \pi$ decays within the inclusive $D_{s}$ sample on real data. The fit results are superimposed.

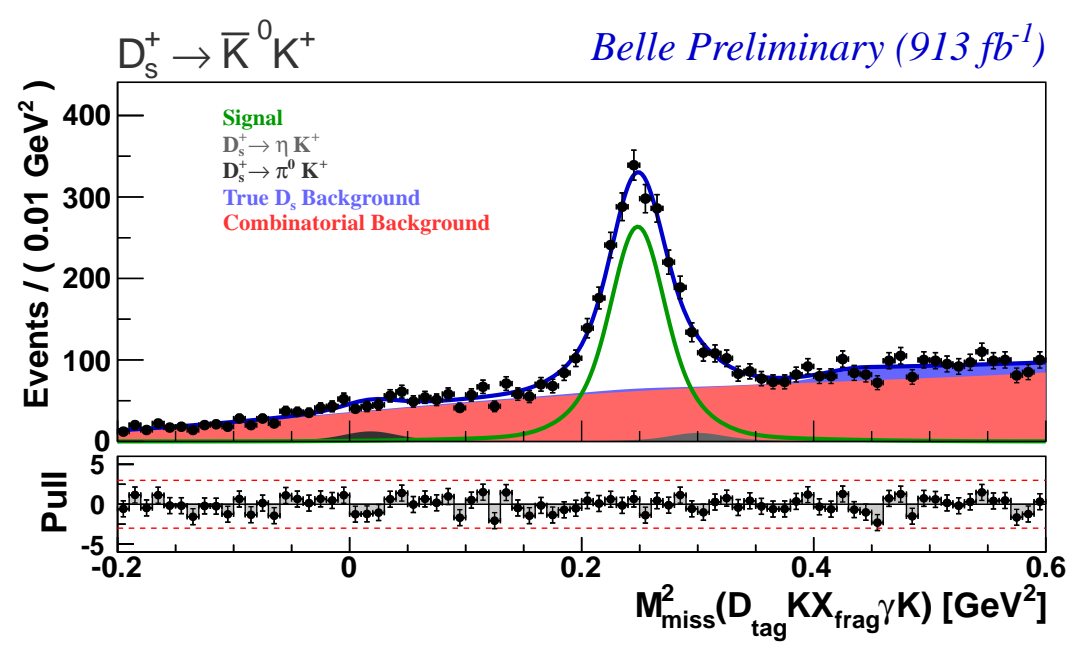

Figure 3: The $M_{\text {miss }}^{2}\left(D_{\text {tag }} K X_{\text {frag }} \gamma K\right)$ distribution of exclusively reconstructed $D_{s}^{+} \rightarrow \bar{K}^{0} K^{+}$decays within the inclusive $D_{s}$ sample obtained on real data with fit results superimposed.

momentum pointing to IP. A simple mass window cut is required for both $\Lambda$ and $\Xi^{-}$. To further suppress the combinatorial background, we require the candidate $\Xi_{c}^{0}$ momentum in the center of mass frame should be greater than $3.0 \mathrm{GeV} / c$ since the charmed particles from $e^{+} e^{-} \rightarrow c \bar{c}$ should be hard.

The measured mass distribution for $\Xi_{c}^{0} \rightarrow \Xi^{-} K^{+}$is shown in Fig. 6. The mass peak is measured to be $2470.6 \pm 1.5 \mathrm{MeV} / c^{2}$ which is in good agreement with the nominal mass of $\Xi_{c}^{0}$ [1]. The feedacross ( $\pi$ to $K$ misidentification) background events from the Cabibbo favored decay $\Xi_{c}^{0} \rightarrow \Xi^{-} \pi^{+}$ form a bump in the high mass region near signal peak. We use the Cabibbo favored decay as the normalization yardstick and obtain $\frac{\mathscr{B}\left(\Xi_{c}^{0} \rightarrow \Xi^{-} K^{+}\right)}{\mathscr{B}\left(\Xi_{c}^{0} \rightarrow \Xi^{-} \pi^{+}\right)}=(2.75 \pm 0.51 \pm 0.25) \times 10^{-2}$.

For the $\Lambda K^{+} K^{-}$sample, we select candidate events in the $\Xi_{c}^{0}$ window and draw the Dalitz plot, 


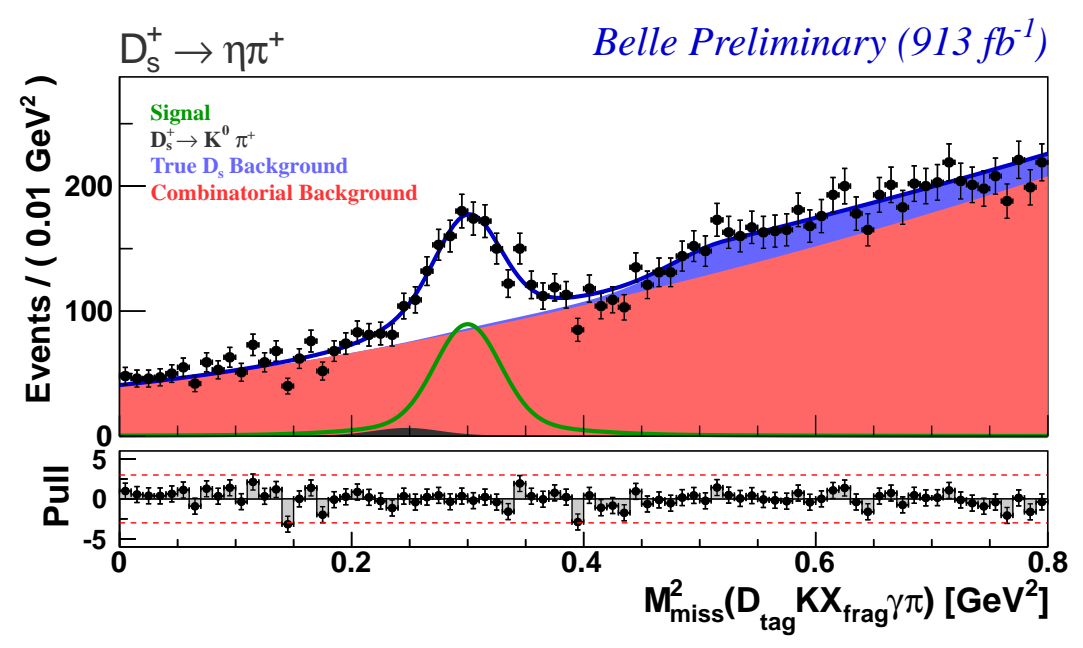

Figure 4: The $M_{\text {miss }}^{2}\left(D_{\text {tag }} K X_{\text {frag }} \gamma \pi\right)$ distribution of exclusively reconstructed $D_{s}^{+} \rightarrow \eta \pi^{+}$decays within the inclusive $D_{s}$ sample obtained on real data with fit results superimposed.

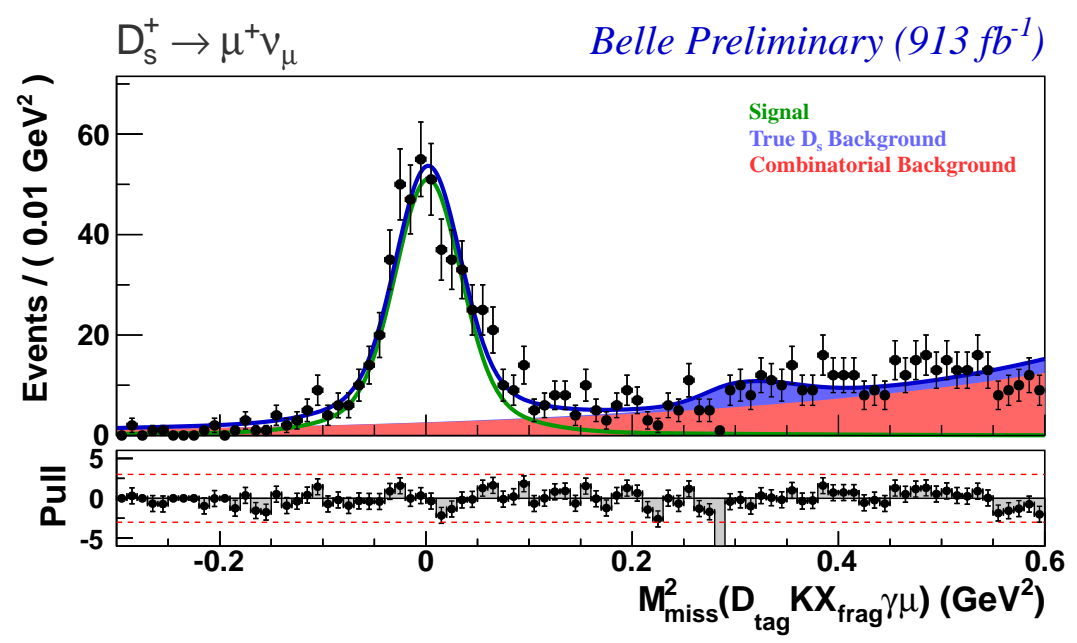

Figure 5: The $M_{\text {miss }}^{2}\left(D_{\text {tag }} K X_{\text {frag }} \gamma \mu\right)$ distribution of exclusively reconstructed $D_{s}^{+} \rightarrow \mu^{+} v_{\mu}$ decays within the inclusive $D_{s}$ sample obtained on real data with fit results superimposed.

$M\left(K^{+} K^{-}\right)^{2}$ vs. $M\left(\Lambda K^{-}\right)^{2}$. The result is shown in Fig. 7. There are clear $\Omega^{-}$and $\phi$ (horizontal) bands. We remove the events in the $\Omega^{-}$band and determine the signal yield for $\Xi_{c}^{0} \rightarrow \Lambda \phi$ by fitting the mass spectrum of $\Lambda \phi$. We obtain $\frac{\mathscr{B}\left(\Xi_{c}^{0} \rightarrow \Lambda \phi\right)}{\mathscr{B}\left(\Xi_{c}^{0} \rightarrow \Xi^{-} \pi^{+}\right)}=(3.43 \pm 0.58 \pm 0.32) \times 10^{-2}$. The above two Cabibbo suppressed decays are observed in the first time and their branching fractions are compatible with each other.

\section{Summary}

The absolute branching fractions of leptonic $D_{s}$ decays $D_{s}^{+} \rightarrow \mu^{+} v$ and $D_{s}^{+} \rightarrow \tau^{+} v$ are measured quite precisely using Belle data alone. The decay constant $f_{D_{s}}$ obtained from the above measurements is consistent with the lattice QCD calculation (within $2 \sigma$ ). The Cabibbo suppressed 


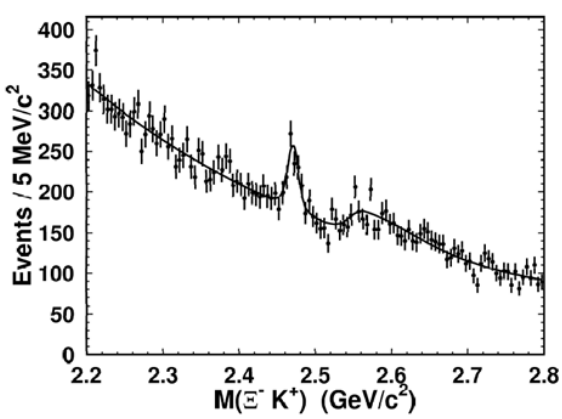

Figure 6: The $M\left(\Xi^{-} K^{+}\right)$distribution with fit results overlaid. The feed-across background events from the Cabibbo favored decay $\Xi_{c}^{0} \rightarrow \Xi^{-} \pi^{+}$form a bump in the high mass region near signal peak.

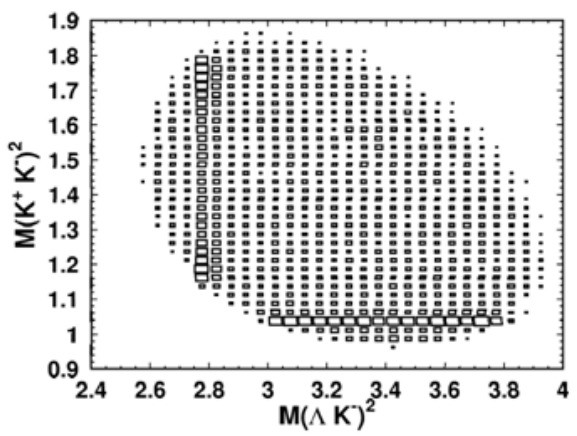

Figure 7: Dalitz plot $M\left(K^{+} K^{-}\right)^{2}$ vs. $M\left(\Lambda K^{-}\right)^{2}$ for events within $\pm 12 \mathrm{MeV}$ mass window around $M\left(\Xi_{c}^{0}\right)$. Clear $\Omega^{-}$(vertical) and $\phi$ (horizontal) bands are seen.

charmed baryon decays, $\Xi_{c}^{0} \rightarrow \Xi^{-} K^{+}$and $\Xi_{c}^{0} \rightarrow \Lambda \phi$, are observed which can offer useful information for the decay dynamics of charmed baryons.

The author wish to thank the KEKB accelerator group for the excellent operation of the KEKB accelerator and the ICHEP2012 organization committee for making such a wonderful conference. This work is supported by the National Science Council of Taiwan under the grant NSC-99-2112M-002-006-MY3.

\section{References}

[1] J. Beringer et al. (Particle Data Group), Phys. Rev. D 86, 010001 (2012).

[2] Throughout this report, inclusion of charge-conjugate modes is always implied unless otherwise stated.

[3] C. T. H. Davies et al. (HPQCD Collaboration), Phys. Rev. D 82, 114504 (2010).

[4] For example, K.K. Sharma and R.C. Verma, Phys. Rev. D 55, 7067 (1997).

[5] A. Abashian et al. (Belle Collaboration), Nucl. Instr. and Meth. A 47 9, 117 (2002).

[6] S. Kurokawa and E. Kikutani, Nucl. Instr. and Meth. A499, 1 (2003) and other papers included in this Volume. 